\title{
An Overview of Consumer Attitudes and Beliefs about Plant Food Supplements
}

\author{
${ }_{5}$ Bernadette Egan, ${ }^{\text {"a }}$ Charo Hodgkins, ${ }^{a}$ Richard Shepherdl, ${ }^{a}$ Ladla Timotijevic, ${ }^{a}$ and Monique Raats ${ }^{\mathrm{a}}$
}

\author{
Received (in $X X X, X X X)$ XthXXXXXXXXX 20XX, Accepted Xth $X X X X X X X X X 20 X X$ \\ DOI: $10.1039 / b 000000 x$
}

The use of dietary supplements is increasing globally and this includes the use of plant food supplements (PFS). A variety of factors may be influencing this increased consumption including the increasing number of older people in society, mistrust in conventional medicine 10 and the perception that natural is healthy. Consumer studies in this area are limited, with a focus on dietary supplements in general, and complicated by the use of certain plant food supplements as herbal medicines. Research indicates that higher use of dietary supplements has been associated with being female, being more educated, having a higher income, being white and being older, however the drivers for consumption of supplements are complex, being influenced by both demographic and health-related factors. The aim of this paper is to provide an overview of current knowledge about the users and the determinants of usage of plant food supplements. With growing 15 consumption of these products, the need for effective risk-benefit assessment becomes ever more important, and an insight into who uses these types of products and why is an important starting point for any future science-based decision making by policy makers, PFS manufacturers and ultimately by consumers themselves.

\section{Introduction}

20 The use of dietary supplements has increased steadily over the years and may in part reflect the growing acceptance and use of a wider range of complementary and alternative therapies ${ }^{1}$. Possible drivers for this increased use include a general dissatisfaction with conventional medicine and a desire for more ${ }_{25}$ control over the decisions relating to one's own health. ${ }^{2,}{ }^{3}$ In the UK, $25 \%$ of the population are reported to use some form of complementary and/or alternative medicine (CAM) and this rises to $50-70 \%$ for populations in France and Germany. ${ }^{4}$ In the United States approximately $38 \%$ of adults are estimated to be using 30 some form of complementary or alternative medicine. ${ }^{5}$

The term "dietary supplement" encompasses a wide range of different substances, including vitamins, minerals, herbal and botanical substances, fish oils, glucosamine, creatine and essential fatty acids. The European Union (EU) Directive on

${ }_{35}$ Food Supplements (2002/46/EC) defines dietary supplements (which include plant food supplements) as:

" ....food stuffs the purpose of which is to supplement the normal diet and which are concentrated sources of nutrients or other substances with a nutritional or physiological effect, alone or in 40 combination, marketed in dose form, namely forms such as capsules, pastilles, tablets, pills and other similar forms, sachets of powder, ampoules of liquids, drop dispensing bottles, and other similar forms of liquids and powders designed to be taken in measured small quantities". ${ }^{6}$ Commonly used plant food 45 supplements include Echinacea, ginkgo biloba, ginseng, green tea extract, St John's Wort and valerian.

The aim of this paper is to provide a brief overview of what is known about users of plant food supplements and the determinants of usage. However, when attempting to review 50 published data on plant food supplements it becomes immediately apparent that the consumer studies performed to date have tended to focus on the use of dietary supplements in general, and not specifically on those that are plant- derived. In the United States for example, plant food supplements often exist as a sub-division 55 of what is known as "Non-Vitamin Non- Mineral (NVNM)" supplements, which encompasses botanicals, protein, amino acids and even shark cartilage. As a consequence, the plant food supplement data within these studies is often masked by this wider dietary supplement category.

${ }_{60}$ In addition plant food supplements are quite often used for medicinal purposes and not specifically as dietary supplements. Whilst the substances themselves are frequently one and the same, it is important to differentiate between the intended uses since this often dictates whether by definition, they are a plant ${ }_{65}$ food supplement or indeed an herbal medicine.

Another area of complexity within the area of plant food supplements is the wide range of different terminology used for these substances; they are interchangeably referred to in the literature as "plant foods", "plant extracts", "botanicals", 70 "herbals" and/or "herbs".

\section{Methods}

A number of electronic databases were searched for relevant papers, including EMBASE, PubMed, PsychInfo, Web of Science and CINAHL (Cumulative Index to Nursing and Allied 75 Health Literature). Manual searches of reference lists and internet searches of grey literature were also used to identify relevant 
articles. The search strategy included references published in English and from all years to 2010. A range of search terms were used, including herbal supplement, plant food supplement, botanicals, consumer, attitudes and beliefs; combinations of terms 5 were also used. Papers were excluded where plants were considered to be a form of herbal medicine.

\section{Who uses Plant Food Supplements?}

Information from academic literature on the prevalence of use of dietary supplements in general, and plant food supplements in 10 particular, comes primarily from the United States where studies use data available from sources such as the National Health and Nutrition Examination Surveys (NHANES), Health and Diet Surveys and National Health Interview Surveys (NHIS). In a recent report based on data from NHANES 2003-2004 and 15 NHANES $2005-2006,49 \%$ of the population of the United States ( $44 \%$ of males, $53 \%$ of females) were estimated to be using dietary supplements. ${ }^{7}$ The use of multivitamins-multiminerals was the most frequently reported $(33 \%)$, with $14 \%$ reporting use of botanical dietary supplements. The majority of people reported 20 taking only one dietary supplement and did so on a daily basis with intake based on use over the past 30 days. Data from the 2007 National Health Interview Survey (NHIS) ${ }^{8}$ indicated that the use of nonvitamin, nonmineral natural products was the most commonly used complementary and alternative therapy among 25 adults, with $17.7 \%$ reporting usage over the past 30 days. Within the top ten natural products a number of plant supplements featured with the most popular being Echinacea, ginseng, ginkgo biloba and garlic supplements.

There is some data available on the increasing use of dietary 30 supplements in a number of European countries 9, 10,11 but less information regarding the prevalence and types of dietary supplements used. The use of dietary supplements in the European Prospective Investigation into Cancer (EPIC) and Nutrition calibration study has been reported. ${ }^{12}$ The EPIC study 35 includes more than half a million participants in 10 European countries: Denmark, France, Germany, Greece, Italy, the Netherlands, Norway, Spain, Sweden and the United Kingdom. ${ }^{13}$ Across all countries the crude mean percentage of supplement users varied from $2 \%$ to $51.8 \%$ for men and from $6.7 \%$ to $65.8 \%$ 40 for women. There was a clear north-south gradient with the highest consumption in Scandinavian countries and the lowest in Mediterranean countries; in all countries use was higher in women than in men. Vitamins, minerals or combinations of them were the predominant types of supplements reported in most

45 countries; herbs/plant -based supplements represented $8-17 \%$ of the products, with no use recorded in Greece (Table 1).

Many studies of dietary supplement use focus on "special populations" such as pre-and post-menopausal women ${ }^{14}$, older adults ${ }^{15,16}$ and individuals with chronic conditions. ${ }^{17}$

${ }_{50}$ One such example is the study examining the prevalence of nonvitamin, nonmineral (NVNM) supplement usage among university students in the US. ${ }^{18}$ Results from that study indicated that $26.3 \%$ of students reported use of NVNM supplements with ginseng, Echinacea, protein powder/amino acids and gingko

55 biloba being most frequently used. Ginseng, Echinacea, protein powders/amino acids, gingko biloba and St John's Wort have similarly been reported as popular supplements in other populations. ${ }^{19,20}$ Characteristics of the users in the study by Perkin and colleagues emerged as: $78.7 \%$ white, more women 60 than men, most rated their health as excellent or good $(82.1 \%)$, $73 \%$ exercised more than three times a month. The reasons for use most noted were to improve energy, to promote weight loss, to burn fat and to supplement an inadequate diet. ${ }^{18}$ In a study with adult members of a large health maintenance organization $32.7 \%$

${ }_{65}$ used at least one NVNM supplement in the 12 months before the survey. ${ }^{21}$ The most frequently used herbs were Echinacea $(14.4 \%)$ and gingko biloba (10.9\%). Use of all NVNM supplements was highest amongst females, 45-64 years of age, white, college graduates and among those with selected health 70 conditions.

In general the following demographics have been associated with a higher use of dietary supplements, being female, education beyond high school, higher income, being white and being older. $^{7,22,23}$ An analysis of data from the 2000 National Health 75 Interview Survey reinforced some of the general demographic and health-related determinants of supplement use but also provided some evidence that determinants may vary by particular supplement. For example, those who were overweight or obese were less likely to use vitamins or minerals (compared to those of 80 non-problematic weight) but this difference was not seen for herbal use. ${ }^{24}$

In the 2002 NHIS CAM survey socioeconomic status was positively associated with herb use, again with higher rates of use among women and middle-aged adults ${ }^{25}$. Herbal consumption ${ }_{85}$ was associated with positive assessments of health status and behaviours such as regular exercise and smoking cessation. There was also a positive association between use of herbal supplements and prescription or over-the -counter (OTC) medications, suggesting the use of supplements as a complement 90 rather than an alternative to conventional medical treatment. This was also reported in a study of consumer spending on herbal products where OTC drug use was positively related to spending on herbals and appeared to complement herb use. ${ }^{26}$

A few studies have attempted to characterise the attitudes 95 towards nutrition and health of supplement users. In a study of the 1946 British birth cohort the underlying diet, health-related characteristics and behaviours of users and non-users of dietary supplements were investigated. ${ }^{27}$ Supplement use was associated with lower BMI, lower waist circumference, higher plasma folate 100 and plasma vitamin B-12 concentrations, non-smoking, participation in physical activity and non manual social class in women and with plasma folate concentrations and participations in physical activity in men

Supplement users were reported to be more likely to have a 105 strong belief in diet-cancer connections than were non- users. ${ }^{28}$ However more often individuals cited health promotion or taking control of their health, rather than disease prevention, as a reason for using supplements or herbs. ${ }^{29,30}$ Supplement use has also been linked to various health-related behaviour patterns; positive 110 lifestyle factors are generally associated with increased supplement use. For example non-smokers were more likely to take supplements than current smokers, as were individuals who consumed no alcohol or moderate amounts of alcohol compared to those who consumed more. ${ }^{31}$ 
5 Table 1. Percentage (\%) of supplement users the previous day, weighted for season and period of the week in the EPIC calibration study and percentage $(\%)$ of herbs/plants used by country and gender ${ }^{12}$

\begin{tabular}{ccccccc}
\hline & Men & & & & Women & \\
\hline & $\mathrm{N}$ & $\%$ & $\%$ herbs/plants & $\mathrm{N}$ & $\%$ & $\%$ herbs/plants \\
\hline Greece & 1311 & 2.0 & 0 & 1373 & 6.7 & 0 \\
\hline Spain & 1777 & 5.9 & 15.0 & 1443 & 12.1 & 11.0 \\
\hline Italy & 1442 & 6.8 & 8.0 & 2511 & 12.6 & 8.0 \\
\hline France & & & & 4735 & 32.4 & 7.0 \\
\hline Germany & 2267 & 20.7 & 16.0 & 2148 & 27.0 & 12.0 \\
\hline The Netherlands & 1024 & 16.0 & 17.0 & 2956 & 32.1 & 18.0 \\
\hline UK & 4.0 & 36.3 & 10.0 & 570 & 47.5 & 10.0 \\
\hline Denmark & 1932 & 51.0 & 10.0 & 1994 & 65.8 & 10.0 \\
\hline Sweden & 2765 & 30.5 & 16.0 & 3285 & 42.4 & 15.0 \\
\hline Norway & & & & 1797 & 60.6 & 8.0 \\
\hline
\end{tabular}

If no figures are indicated no strata were sampled.

\section{${ }_{10}$ 4. Reasons for using Plant Food Supplements}

There have been number of studies of the factors associated with use of plant food supplements, using both qualitative and quantitative research methods. One study of factors that influence the consumption of dietary supplements explored the

15 reasons for purchase and use of multivitamins, concentrates and herbs/flowers and roots among dietary supplement users, aged 18-83, from a medium-sized Southern U.S city. ${ }^{32}$ Qualitative indepth interviews were conducted with ten consumers of dietary supplements and two pharmacists. Subsequently 900 20 questionnaires were distributed through pharmacies, of which 225 were completed and returned. The interviews revealed both physical and psychological issues motivating supplement consumption, with consumers citing reasons such as curing an ailment, preventing chronic disease, gaining "peace of mind",

${ }_{25}$ supplementing a poor diet or an unhealthy lifestyle and saving money on medical care. Consumers perceive that supplements are a form of proactive insurance that gives them a degree of control over their health. Results from the questionnaire survey shed light on differences in behaviour according to the type of dietary 30 supplement consumed (multivitamin; multivitamin and concentrates and multivitamin, concentrates and herbs/flowers/roots). Those who consume a diverse set of products have stronger beliefs in the benefits of dietary supplements, perceive more risk in using vitamins and are more 35 strongly influenced by the media.

An ongoing survey by telephone of a random sample of the noninstitutionalised US population for use of medications revealed that $14 \%$ had taken at least one herbal/supplement in the preceding week with ginseng and ginkgo biloba being the most 40 popular. The most frequently reported reasons for use were "health/good for you", arthritis, memory improvement, energy and immune booster. ${ }^{19}$

A study of the use, attitudes and knowledge of herbal products and dietary supplements amongst 267 older adults in Kansas 45 revealed that fifty-six $(21 \%)$ respondents were currently taking at least one herbal product or dietary supplement, with glucosamine and garlic being the most frequently used, closely followed by Echinacea and ginkgo biloba. ${ }^{15}$ The most common reasons reported for using these products included helping to manage 50 arthritis, preventing or managing colds or improving memory. However the most significant predictor of use was an interest in the "general health purposes" of these substances.

Previous work suggests that supplement users tend to have healthier lifestyle profiles than non-users ${ }^{33}$ and that supplement ${ }_{55}$ taking may be viewed as an "insurance" against possible ill health. ${ }^{34}$ Overall supplement users tended to differ from nonusers on a range of health-related behaviours. In studies exploring the use of dietary supplements in a cohort of women the reasons for consumption emerge as a complex mix of factors. ${ }^{35,36}$ These ${ }_{60}$ studies used the Theory of Planned Behaviour ${ }^{37}$ to investigate the use of herbals and dietary supplements in women. Intentions were a major predictor of supplement use; health value and susceptibility to illness were also significant predictors of dietary supplement use $(82.9 \%$ of respondents were correctly classified ${ }_{65}$ as users or non users). Supplement users believed more strongly than non-users that taking dietary supplements would stop them getting ill and help them to be healthy.

\section{Perceived benefits and risks}

The popularity and increased use of dietary supplements have led 70 to concerns about their safety and possible health risks, particularly as most patients are reluctant to share information on their usage with their healthcare providers ${ }^{3,38,39}$. Problems can arise in a number of ways including improper use of the product, product defects, poor quality, questionable purity strength and /or 75 composition. Botanical supplements are frequently criticised for poorly proven efficacy and safety, lack of standardization and quality standards and potential interactions with prescribed drugs .$^{40}$ Regulations for dietary supplements are very different from those for prescription and over-the -counter drugs. Use of plant ${ }_{80}$ food supplements in the USA increased dramatically after the passage of the Dietary Supplement and Health Education Act of 1994 (DSHEA) $^{41}$ whereby botanicals can be marketed with few regulatory impediments, providing that disease prevention, curing 
or detection claims are not made. Instead, so called structurefunction claims on products can relate to enhancing or maintaining normal physiological functions of the human body Information from the 2002 Health and Diet Survey revealed that 5 of the $73 \%$ of US adults who had used a dietary supplement in the past year, $4 \%$ reported adverse effects. ${ }^{23}$ There was a higher proportion of concurrent use of dietary supplements with prescription drugs among users with adverse effects compared with users without adverse effects. Evidence suggests that users

10 of dietary supplements tend to be less likely to reveal their use of dietary supplements to health care professionals than their use of medication. Additional findings from the Alternative Health supplement of the 2002 National Health Interview Survey (NHIS) revealed that roughly two thirds of adults using commonly

15 consumed herbs (except Echinacea) did not do so in accordance with evidence-based indications. ${ }^{42}$

An examination of the patterns of nonvitamin dietary supplement (NVDS) use among adult prescription users indicated that $21 \%$ of users report using NVDS in the prior 12 months. ${ }^{43}$ Of those $69 \%$

20 did not discuss this use with a medical practitioner. The most commonly used supplements included Echinacea, ginseng, ginkgo, garlic and glucosamine. Prescription medication users with menopause and chronic gastrointestinal disorders had the highest rates of NVDS use (33\% and $28 \%$ ). Factors associated 25 with increased use of NVDS included being female, being Hispanic, having more years of education, living in the western US, lacking medical insurance and having chronic conditions. A survey regarding the safety and testing of herbal products and dietary supplements revealed a number of misperceptions among 30 the survey respondents. ${ }^{15}$ Two thirds of all respondents falsely believed that such products pose no risk to the general population and the majority incorrectly thought that the Food and Drug administration test and regulate these products.

35 An investigation of people's views of the efficacy and specific risks of herbal, over-the counter (OTC) conventional and prescribed conventional medicines led to the conclusion that people need clear information about the risks and benefits of herbal supplements if they are to use such products safely and ${ }_{40}$ effectively. ${ }^{44}$ Both drug and supplement marketing may rely on scientific jargon to persuade consumers and may claim to promote health or prevent disease in ways that may confuse the consumer. ${ }^{45}$ Health claims have the potential to be an important communication tool for consumers. In the context of EU

45 Regulation 1924/2006 health claims are those that state, suggest or imply a relationship between a food or food category and health; with a requirement that the claim is scientifically proven. ${ }^{46}$ Examples include function claims, reduction of disease risk claims or claims referring to the growth and development of ${ }_{50}$ children. ${ }^{47} \mathrm{~A}$ number of studies have shown that consumers do not always understand nutrition and health claims as they are intended. ${ }^{48,49}$

Findings from in depth interviews with dietary supplement users in the US indicated that a primary source of influence for some is ${ }_{55}$ their physician; health professionals may also influence use. ${ }^{32}$ Family, mass media, particularly magazine news and television stories, also serve as sources that convince consumers of the need to take supplements.

\section{Attitudes and beliefs of health professionals}

${ }_{60}$ Several studies have been conducted among healthcare professionals concerning their personal use, beliefs and recommendations regarding dietary supplements. ${ }^{50,51,52}$ Most of the studies have focused on dieticians who are regarded as experts on healthy diet and lifestyle.

${ }_{65}$ Understanding their views is considered important as dieticians may be in a position to offer science-based information regarding claims made by the supplement industry and to provide advice that may reduce drug interactions and contraindications.

In a cross-sectional pilot study examining the recommending 70 practices, personal use and beliefs of dieticians in the Netherlands $64 \%$ reported using dietary supplements themselves for prevention of a health condition, $60 \%$ for treatment of a health condition and $44 \%$ for enhancing physical or mental performance. ${ }^{52}$ In turn $71 \%$ indicated recommending supplements 75 for prevention, $82 \%$ for treatment of a health condition and $43 \%$ for enhancement of performance. Dietary supplements were considered at least moderately safe by $94 \%$ of dieticians, $75 \%$ considered supplements moderately effective in preventing a health condition, $91 \%$ for treatment of a health condition and $8059 \%$ for performance enhancement.

In a study with California registered dieticians participants were asked if they used or recommended both nutrient and herbalbased dietary supplements and to list up to five supplements and the primary reason for using or recommending them. ${ }^{53}$ Overall ${ }_{85} 83 \%$ agreed that they are frequently asked questions about dietary supplements; $89 \%$ believe they convey factual dietary supplement information effectively, 95\% believed they should learn more. A majority of the sample reported using (69\%) and recommending (74\%) nutrient-based dietary supplements, 90 whereas fewer reported using (18\%) or recommending (13\%) herbal -based supplements. Registered dieticians who used supplements were significantly more likely to recommend them to their clients. The most common herb was Echinacea and the most common reasons for using and recommending herbal-based 95 supplements were for immune enhancement, to treat menopause and to improve memory and prostate health. In a similar study in Oregon, dieticians considered themselves to be knowledgeable about functional foods and nutrient supplements- more than $80 \%$ were confident of the effectiveness for prevention of illness and 100 treatment of chronic illness, $89 \%$ confident of their safety. In contrast fewer than $75 \%$ considered herbs to be safe and only $50 \%$ were confident of the effectiveness of herbs. Only $10 \%$ were knowledgeable about herbs for prevention and treatment of illness; fewer than $42 \%$ reported use of herbs. ${ }^{54}$

105 In a study focusing solely on herbal supplements the majority of Massachusetts registered dieticians lacked herbal supplement familiarity; $73 \%$ perceived themselves to have little or no knowledge. $37 \%$ reported using herbs and $22 \%$ recommending herbs to clients in the past year. ${ }^{55}$

\section{Summary and Conclusions}

The use of plant food supplements is increasing globally with demand driven by a variety of factors including an ageing population, a growing tendency to self-medicate, mistrust in 
conventional medical practice and the perception that natural is healthy and plant products are safe. Consumers regularly face decisions about engaging in healthy lifestyles and the market is flooded with products that are meant to help them achieve that ${ }_{5}$ goal. This market includes health remedies such as drugs and supplements.

Data on actual consumers of plant food supplements are limited as intake surveys most often refer to the broader category of dietary supplements, with a focus on the intake of vitamins and 10 minerals. Although results from the United States indicate that in general, PFS use is associated with being female, middle-aged, having better education and a higher income the drivers for consumption of supplements are often complex, being influenced by a combination of demographic and health-related factors.

${ }_{15}$ Consumer's motives for using PFS vary and include the maintenance of overall general health, prevention of disease, a means of compensating for an unhealthy lifestyle or treatment for specific diseases and conditions.

Whilst a number of studies have highlighted safety issues 20 associated with the intake of plant food supplements, the regulation of supplements is much less stringent than that of prescribed or over-the-counter medicines. In addition, the majority of consumers do not disclose their use of such products to health professionals and therefore the occurrence of adverse ${ }_{25}$ effects is thought to be under-reported.

With growing consumption of these products, the need for effective risk-benefit assessment becomes ever more important, and an insight into who uses these types of products and their motives for doing so is an important starting point for any future 30 science-based decision making by policy makers, PFS manufacturers and ultimately by consumers themselves. Furthermore we need to have a better understanding of the health-related behaviours of those consuming supplements in order to ensure that those who might benefit most from 35 supplement intake do so.

\section{Acknowledgement}

The research leading to these results has received funding from the European Community's Seventh Framework Programme (FP7/2007-2013) under grant agreement $\mathrm{n}^{\circ}$ 245199. It has been 40 carried out within the PlantLIBRA project (website: www.plantlibra.eu). This report does not necessarily reflect the Commission views or its future policy on this area.

\section{Notes and references}

${ }^{a}$ Food, Consumer Behaviour and Health Research Centre, Department 45 of Psychology, Faculty of Arts and Human Sciences, University of Surrey, Guildford, Surrey, UK

Fax: +44(0)1483682913; Tel: +44(0)1483682888;

E-mail:m.egan@surrey.ac.uk

1. F. Bishop and G. Lewith, Who uses CAM? A narrative review of demographic characterisitics and health factors associated with CAM use. eCAM, 2008 7(1):11-28.

2. J. Caulfield, The psychological aspects of complementary and alternative medicine. Pharmacotherapy, 2000, 20, 1289-1294

3. D. Eisenberg, R. Davis, S. Ettner, S. Appel, S. Wilkey, M.Van Rompey and R. Kessler, Trends in alternative medicine use in

the United States, 1990-1997 J. Am. Med. Assoc., 1998280 , 1569-1575

. Mehta, P. Gardiner, R. Phillips and E. McCarthy, Herbal and dietary supplement disclosure to health care providers by individuals with chronic conditions. J. Altern. Compl. Med., 2008, 14 (10), 1263-1269. 
18. J. Perkin, W. Wilson, K. Schuster, J. Rodriguez and A. AllenChabot, Prevalence of nonvitamion, nonmineral supplement usage among university students. J. Am. Diet. Assoc. , 2002, 102 (3) 412-414.

19. D. Kaufman, J. Kelly, L. Rosenberg, T. Anderson and A. Mitchell, Recent patterns of medication use in the ambulatory adult population of the United States. J. Am. Med. Assoc., 2002, 287 (3), 337-344.

20. J. Kennedy, Herb and Supplements use in the US adult population. Clin. Ther., 2005, 27(11), 1847-1858.

21. D. Schaffer, N. Gordon, C. Jensen and A. Avins, Nonvitamin, nonmineral supplement use over a 12-month period by adult members of a large health maintenance organization. J. Am. Diet. Assoc. 2003, 103 (11),1500-1505.

22. R. Nayga and D. Reed, Factors associated with the intake of dietary supplements. Fam. Econ. Nutr. Rev., 1999, 12(1), 4347.

23. B. Timbo, M. Ross, P. McCarthy and C.Lin, Dietary supplements in a National Survey: Prevalence of use and reports of adverse events. J. Am. Diet. Assoc., 2006, 106:19661974.

24. D. Fennell, Determinants of supplement usage. Prev. Med. 2004, 39, 932-939.

25. National Centre for Health Statisitics 2002 National Health Interview Survey (NHIS). Public use data release Hyatsville, Md: USA Dept of Health and Human Services, Centers for Disease Control and Prevention, National Centre for Health Statisitics, 2003 Available at ftp://ftp.cdc.gov/pub/Health_Statistics/NCHS/Dataset_Docum entation/NHIS/2002/srvydesc.pdf. ASccessed April 27th 2011.

26. F. Alkhateeb, W. Doucette and J. Ganther-Urmie, Influences on consumer spending for herbal products, Res. Soc. Admin. Pharm., 2006, 2, 254-265.

27. S. McNaughton, G. Mishra, A. Paul, C. Prynne and M Wadsworth, Supplement use is associated with health status and health-related behaviors in the 1946 British Birth Cohort. J. Nutr., 2005, 135, 1782-1789

28. R. Patterson, M. Neuhouser, E. White, J. Hunt and A. Kristal, Cancer-related behaviour of vitamin supplement users. Cancer Epidem. Biomar. Prev. , 1998, 7:79-81.

29. D. Hensrud, D. Engle and S. Scheitel, Under-reporting the use of dietary supplements and non-prescription medications among patients undergoing a periodic health examination, Mayo Clin. Proc., 1999, 74, 443-447.

30. HG Schutz, M Read, R Bendel, VS Bhalla, I Harrill, JE Monagle, ET Sheehan and BR Standal, Food supplement usage in seven Western states, Am J Clin Nutr.1982 36 (5) 897-901.

31. J. Greger, Dietary Supplement Use: Consumer characteristics and interests. J. Nutr., 2001, 131,1339S-1343S.

32. C. Peters, J.Shelton and P. Sharma, An investigation of factors that influence the consumption of dietary supplements. Health Marketing Ouality, 2003, 12,(1/2:)113-134.

33. S. Kirk, J. Cade, J. Barrett and M. Conner, Diet and lifestyle characteristics associated with dietary supplement use in women. Public Health Nutr. , 1999, 2, 69-73.

34. S, Kirk, A. Woodhouse and M. Conner, Beliefs, attitudes and behaviour in relation to supplement use in the UK Women's Cohort Study (UKWCS). Proc. Nutr. Soc., 1998, 57: 54A

35. M. Conner, S. Kirk, J. Cade and J. Barrett, Why do women use dietary supplements? The use of the theory of planned behaviour to explore beliefs about their use. Soc. Sci. Med. 2001 52, 621-633

36. M. Conner, S. Kirk, J. Cade and J. Barrett, Environmental influences: factors influencing a woman's decision to use dietary supplements, J. Nutr. 2003, 133, 1978S-1982S.

37. I. Ajzen, The theory of planned behaviour, Org. Beh. Hum. Dec. Proc., 1991, 50, 179-211

38. D.Eisenberg, R. Kessler, C.Foster, F. Norlock, D. Calkins and $\mathrm{T}$.Delblanco, Unconventional medicine in the United
States: Prevalence, costs and patterns of use, New Engl. J. Med, 1993, 328, 246-252

39. N. Elder, A. Gillcrist and R. Mionz, Use of alternative health care by family practice patients. Arch. Fam. Med., 1997, 6 , 181-184.

40. I. Raskin, D. Ribnicky, S. Komarnytsky, N. Ilic, A. Poulev, N, Borisjuk, A. Brinker, D. Moreno , C. Ripoll , N. Yakoby, J. O'Neal , T. Cornwell , I.Pastor and B. Fridlender, Plants and human health in the twenty-first century. TRENDS Biotechnol. 2002, 20(12), 522-531

41. Dietary Supplement and Health Education Act of 1994. PUBL No.103-417, 21 USC 3419 (1994)

42. A. Bardia, N. Nisly, M. Zimmerman, B. Gryzlak and R. Wallace, Use of herbs among adults based on evidence-based indications: findings from the National Health Interview Survey, Mayo Clin. Proc, 2007, 82(5) 561-566.

43. P. Gardiner, R. Graham, A. Legedza, D. Eisenberg and R. Phillips, Factors associated with dietary supplement use among prescription medication users. Arch Intern. Med. 2006 , 166, 1968-1974.

44. N. Lynch and D. Berry, Differences in perceived risks and benefits of herbal, over -the- counter conventional, and prescribed conventional medicines, and the implications of this for safe and effective use of herbal products. Compl. Ther. Med., 2007, 15, 84-91.

45. L. Bolton, A. Reed, K. Volpp and K. Armstrong, How does drug and supplement marketing affect a healthy lifestyle? $J$ Consum. Res., 2008, 34,713-726.

46. EC Regulation No 1924/2006 (2007) EC regulation No $1924 / 2006$ on nutrition and health claims made on foods Published in the official Journal of the European Union. L12/3-L12/18. Available at: http://eurlex.europa.eu/LexUriServ/LexUriServ.do?uri=OJ:L:2 007:012:0003:0018:EN:pdf.

47. H. Verhagen, E. Vos, S. Franci, M. Heinonen and H. van Loveren, Status of nutrition and health claims in Europe. Arch Biochem. Biophys., 2010, 501:6-15.

48. T. Bech-Larsen and K.Grunert, The perceived healthiness of functional foods: A conjoint study of Danish, Finnish and American consumers' perception of functional foods, Appetite, 2003, 40,9-14.

49. UK Food Standards Agency,Health claims on food packaging: consumer - related qualitative research (Forum Qualitative Reportf016) 2002. Available from :http://www.food.gov.uk/multimedia/pdfs/healthclaims.pdf.

50. A. Dickson, N. Boyon and A. Shao, Physicians and nurses use and recommend dietary supplements: report of a survey, Nutr. J., 2009, 8, 29.

51. V. Lederman, F. Huffman and E. Enrione, Practices, attitudes and beliefs regarding dietary supplements among Florida's dietitians and nurses. J. Diet. Suppl., 2009, 6, 124-142

52. A.Ten Hove, Dietitians in the Netherlands and dietary supplements: practices, personal use and beliefs. Int. J.Nutr. Met.,2011, 3(2): 11-16.

53. C. Hetherwick, M. Neyman, M.Morris and K. Silliman, Perceived knowledge, attitudes and practices of California registered dietitians regarding dietary supplements. $J$. Am. Diet Assoc., 2006, 106,438-442.

54. Y, Lee, C. Georgiou and C. Raab, The knowledge, attitudes and practices of dietitians licensed in Oregon regarding functional foods, nutrient supplements and herbs as complementary medicine, J. Am. Diet. Assoc., 2000, 100: 543-548.

55. L. Cashman, J. Burns, I. Otieno and T. Fung, Massachusetts registered dietitians' knowledge, attitudes, opinions, personal use and recommendations to clients about herbal supplements. J. Altern. Complem. Med, 2003, 9, 735-746. 